Actitudes de los estudiantes de psicología frente a la investigación

Attitudes of students of psychology in front of research

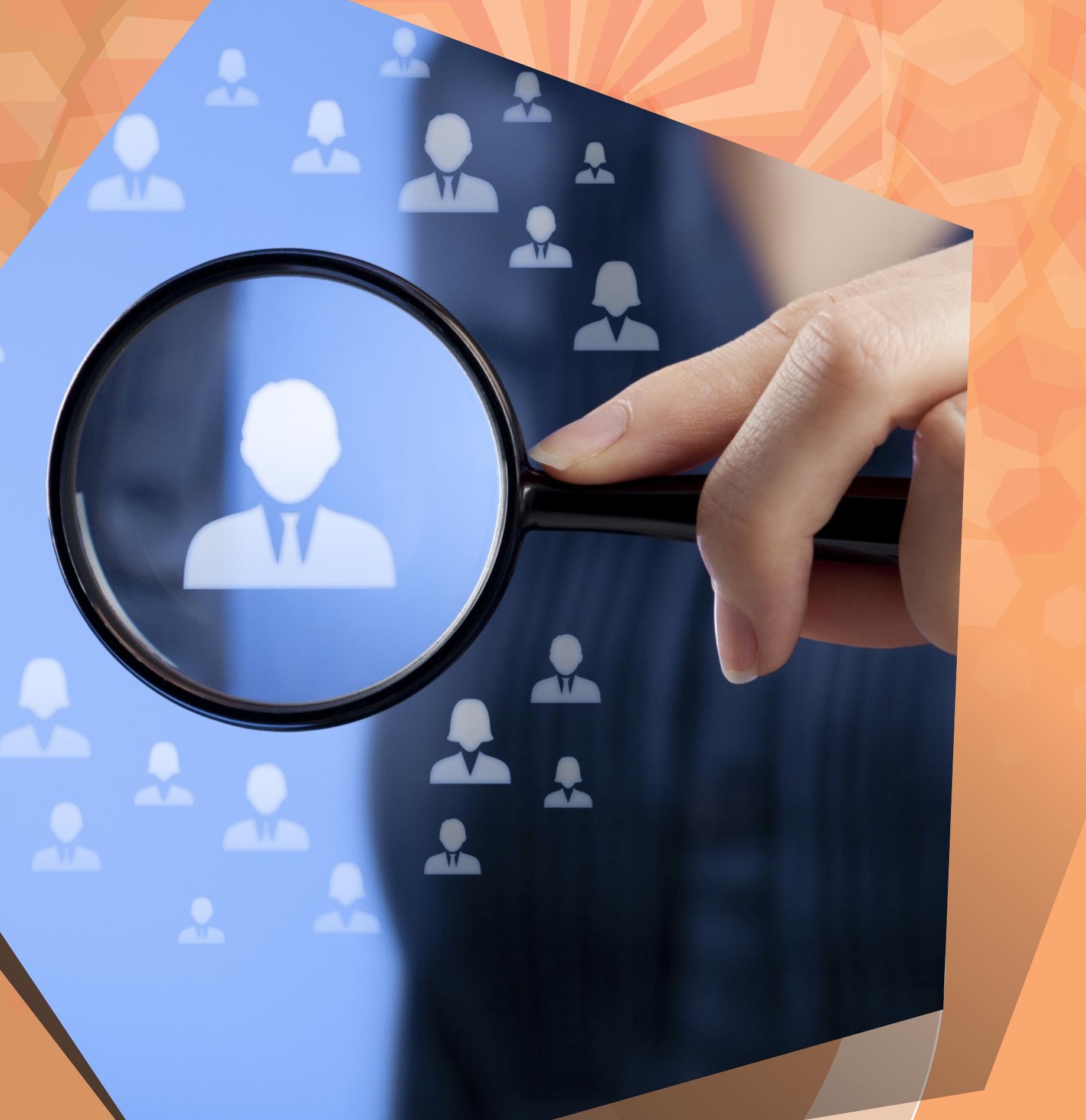




\title{
Actitudes de los estudiantes de psicología frente a la investigación ${ }^{1}$. Attitudes of students of psychology in front of research
}

\author{
Gustavo Alfonso Villamizar Acevedo². Karol Núñez Lobo³. Jenny Andrea Rolón \\ Arias ${ }^{4}$. \\ Universidad Pontificia Bolivariana, Seccional Bucaramanga, Colombia.
}

Artículo recibido en marzo de 2016; artículo aceptado en mayo de 2016

Citación del artículo: Villamizar, G.; Núñez, K. \&Rolón, J. (2016).Actitudes de los estudiantes de psicología frente a la investigación. I+D Revista de Investigaciones, 7(1), 49-60.

\section{Resumen}

Esta investigación tuvo como objetivo identificar las actitudes frente a la investigación en estudiantes de Psicología de una universidad privada de la ciudad de Bucaramanga y la incidencia en ella de variables como: edad, género y nivel de formación. Para alcanzar lo propuesto se realizó una investigación cuantitativa de carácter descriptivo, tipo transversal, que permitió analizar y comparar la información recogida en estudiantes de diversos niveles de formación. Para obtener los datosse aplicó a 233 estudiantes de I a X semestre, entre 15 y 31 años $(\bar{X}=19,8)$ el cuestionario Actitudes hacia la investigación científica. El índice de confiabilidad del cuestionario fue de 0.90. La investigación abarcó los tres componentes de las actitudes, los datos muestran que el $60 \%$ de los estudiantes puntúan muy alto en el componente cognitivo, el $67.8 \%$ puntúanalta en el componente afectiva y el $69.5 \%$ puntúan alto en la conductual. Los resultados no muestran diferencias por género, pero si por edad y nivel de formación. A partir de este último dato, se infiriere que la formación investigativa permite la apropiación de conocimientos y consolidación de herramientas importantes para el ejercicio investigativo. Es de resaltar que no se encontraron puntuaciones muy baja, y que bajas solo se obtuvieron hasta mitad de carrera, deduciéndose que a medida que los estudiantes ingresan al ciclo profesional, donde se conocen los campos de aplicación profesional y se inicia el proceso de práctica, hay una actitud más favorable hacia el ejercicio investigativo al integrar la teoría con la práctica.

Palabras Claves: Actitud, investigación, componentes, cuestionario, estudiantes.

\section{Abstract}

The aim of this investigation was to identify the attitudes of the students of Psychology of a private university towards research. The study also measured the participants commitment towards research taking into account variables such as age, gender and academic level. A descriptive quantitative research was implemented in order to reach the goal of the investigation. A questionnaire entitled "Attitudes towards scientific research" was applied for the purpose of collecting information. This tool was applied to 233 students from first to fifth semester, with ages was 15 to 31 years old. This information was collected, compared and analyzed. The degree of reliability obtained from the questionnaire was 0.90 . This investigation focused on three aspects of students attitudes. The first aspect was the cognitive dimension. The results indicate a high level of the students attitude towards this dimension $60 \%$. The second aspect was the affective dimension, giving as a result $67,8 \%$ And the third aspect was the behavioral dimension, which shows 69.5. The results obtained do not indicate differences between gender, but suggest that age and academic level have an effect on research. Based on this claim, it can be

\footnotetext{
${ }^{1}$ Artículo empírico, enfoque cuantitativo, desarrollado en el Grupo de Investigación Análisis y Transformación Psicosocial Universidad Pontificia Bolivariana Bucaramanga.

${ }^{2}$ Psicólogo, Universidad Nacional de Colombia, Doctor en Educación, Universidad Pedagógica Experimental Libertador. Docente investigador del Grupo Análisis y Transformación Psicosocial. Universidad Pontificia Bolivariana Bucaramanga (Colombia). Autopista Piedecuesta Km 7. www.upb.edu.co.

${ }^{3}$ Psicóloga. Universidad Pontificia Bolivariana Bucaramanga (Colombia). Autopista Piedecuesta Km 7.karolnunezl@gmail.com

${ }^{4}$ Psicóloga. Universidad Pontificia Bolivariana Bucaramanga (Colombia). Autopista Piedecuesta Km 7. jenny.andrea.rolon@gmail.com
} 
concluded that academic training on research provides the knowledge and tools vital for the success of research. It can be emphasized that answers indicating "very low" were not found in the questionnaire. Responses specifying "low" were found only up to the middle point of the career. It can be inferred from this that as soon as the students enroll in the "Professional cycle" there is a tendency to undertake research work and the students can put the theory into practice.

Key Words: Attitude, research, components, questionnaire, students.

\section{Introducción}

Una de las tareas de la universidad es favorecer el encuentro del estudiante con la ciencia, despertando su interés no sólo por adquirir información sino también por producir conocimiento (Valente \& Viana, 2007).Para construir conocimiento es indispensable que los estudiantes desarrollen actitudes positivas frente a la investigación, ya que investigar promueve una visión crítica y permite responder a las exigencias del medio, por lo cual desde los diversos escenarios académicos se debe, no solo promover el interés por la investigación, sino también facilitar al estudiante la comprensión de su papel frente así mismo, frente a la universidad y la sociedad.

Lograr que los estudiantes se inquieten por hacer ciencia es lo que se conoce como Formación Investigativa, la cual "puede ser entendida como aquella que desarrolla la cultura investigativa y el pensamiento crítico y autónomo que permite a estudiantes y profesores acceder a los nuevos desarrollos del conocimiento" (Jiménez, 2006, p. 37). Pero el interés de los estudiantes por construir conocimiento depende de sus posturas actitudinales frente a su proceso de investigacióndado que es en ese proceso donde él adquiere saberes que le permitirán conocer métodos para acceder al conocimiento y posteriormente hacer aportes a las ciencias desde su campo de desarrollo profesional.(Nobigrot, Kleinman, Nobigro-Streimbleinsky \& Galván- Huerta, 1995).

La importancia de cultivar la capacidad investigativa desde la formación profesionalfue analizada por Piña y
León (2013), a quienes el 58,5\% de sus encuestados les manifestaron estar "poco motivado" hacia el ejercicio investigativo desarrollado desde la universidad y sólo $32,3 \%$ valoran los conocimientos derivados de la línea de metodología de la investigación. Rojas, Méndez y Rodríguez (2012), trabajando con estudiantes de cinco universidad de Bucaramanga evidenciaronuna tendencia positiva hacia la investigación aun cuando en las instituciones no haya condiciones que favorecen el ejercicio investigativo. Díaz, Manrique, Galán y Apolaya (2008) así como Rojas (2009) y Kaan y Göksu (2010), encontraronen universitarios actitud positiva hacia la investigación.

Teniendo en cuenta que la Facultad de Psicología donde se investigó enfatiza en la investigación, se evaluaron las actitudes de los estudiantes de psicología hacia este proceso, con el interés de brindar a la Facultad evidencias sobre lo que piensan y sienten frente a la investigación. A partir de estos presupuestos se planteó la siguiente pregunta ¿Cuáles son las actitudes que han formado los estudiantes de Psicología de una universidad privada de la ciudad de Bucaramanga respecto a la investigación?

\section{Tipo de estudio.}

\section{Metodología}

Esta investigación es cuantitativa de carácter descriptivo, tipo transversal, ya que permite analizar y comparar la información recogida en estudiantes de diversos niveles de formación.

\section{Participantes.}

La muestra estuvo conformada por 233 estudiantes, 204 mujeres y 41 hombres, que se encontraban matriculados en los 10 semestres de formación en el primer semestre del 2014, en el centro de estudios donde se efectuó la investigación. Como criterio de inclusión se consideró el que tuvieran matricula académica y administrativa $\mathrm{y}$ accedieran libremente a participar previa aceptación del consentimiento informado. La selección de los participantes se hizo por medio de un muestreo aleatorio y estratificado.

La descripción de los participantes según nivel de formación y género se presenta en la tabla 1. 
Tabla 1. Descripción de los participantes, según semestre y género.

\begin{tabular}{ccccc}
\hline $\begin{array}{c}\text { Nivel de } \\
\text { formación }\end{array}$ & Población & Participantes & Mujeres & Hombres \\
\hline 1 & 111 & 45 & 38 & 7 \\
2 & 45 & 19 & 16 & 3 \\
3 & 81 & 33 & 29 & 4 \\
4 & 52 & 21 & 16 & 5 \\
5 & 81 & 32 & 27 & 5 \\
6 & 31 & 13 & 10 & 3 \\
7 & 65 & 27 & 23 & 4 \\
8 & 36 & 15 & 11 & 4 \\
9 & 36 & 15 & 13 & 2 \\
10 & 13 & 13 & 10 & 3 \\
TOTAL & 551 & 233 & 193 & 40 \\
PORCENTAJE & $100 \%$ & $42.28 \%$ & $82.83 \%$ & $17.17 \%$ \\
\hline
\end{tabular}

Fuente: Los autores

Los datos nos muestran que el grupo más numeroso corresponde a primer semestre, y que la cantidad de mujeres es mayor que los hombres, la correspondencia es de cada diez estudiantes ocho son mujeres.

Tabla 2.Distribución de los estudiantes por género y edad

\begin{tabular}{|c|c|c|c|c|c|c|}
\hline & & & \multicolumn{3}{|c|}{ Edad } & \multirow[b]{2}{*}{ Total } \\
\hline & & & $15-20$ & $21-25$ & $26-31$ & \\
\hline \multirow{4}{*}{ Género } & \multirow{2}{*}{ Femenino } & Estudiantes & 129 & 62 & 2 & 193 \\
\hline & & $\%$ dentro de Género & $66.8 \%$ & $32.1 \%$ & $1 \%$ & $100,0 \%$ \\
\hline & \multirow{2}{*}{ Masculino } & Estudiantes & 25 & 14 & 1 & 40 \\
\hline & & $\%$ dentro de Género & $62.5 \%$ & $35 \%$ & $2.5 \%$ & $100,0 \%$ \\
\hline \multirow{2}{*}{ Total } & & Estudiantes & 154 & 76 & 3 & 233 \\
\hline & & $\%$ dentro de Género & $66,1 \%$ & $32,6 \%$ & $1,3 \%$ & $100,0 \%$ \\
\hline
\end{tabular}

En cuanto a la distribución de los participantes según género y edad, se muestra en la tabla 2.

Fuente: Los autores

La tabla muestra una mayor concentración de estudiantes en el rango de edad correspondiente a 15-20 años, lo cual se relaciona con su nivel de escolaridad, ya que mayor número de los participantes se encuentran matriculados entre I y V semestre.

\section{Instrumentos.}

Como instrumento se utilizó el cuestionario de Aldana y Joya (2011) Actitudes hacia la investigación científica en docentes de metodología de la investigación, el cual consta de 61 afirmaciones (Ver anexo). El formato original la prueba fue sometido a validez de contenido así como a análisis de confiabilidad, este último a través del alfa Cronbach, cuyo resultado 0.97 muestra un alto nivel de confiabilidad.
Considerando que hubo necesidad de modificar algunas afirmaciones de la prueba, debido al cambio de la población a la cual se dirigía, de maestros se pasó a estudiantes, se sometió la prueba modificada al juicio de dos expertos, los cuáles bajo su análisis aprobaron su contenido. La confiabilidad de la prueba adaptada se midió a través del Alfa de Cronbach. Los resultados de la confiabilidad para la totalidad de la prueba fue de 0.90 , por componentes los resultados fueron, cognitivo: 0.85 ; afectivo y conductual 0.79. Datos que muestran la prueba como confiable.

\section{Procedimiento.}

Inicialmente se seleccionó el instrumento a utilizar, después de revisar varios se decidió trabajar con el elaborado por Aldana y Joya (20111), pero teniendo en 
cuenta que originalmente estaba destinado para docentes de realizaron las adecuaciones pertinentes, de forma tal que pudiera ser respondido por estudiantes, luego de ello se sometió a análisis de jueces. Posteriormente se procedió a seleccionar los participantes, para ello se realizó un muestreo estratificado. Una vez seleccionado los participantes se efectuaron tres reuniones con ellos, donde se les comentó sobre la investigación, se les solicitó su colaboración y a los que aceptaron participar se les entregó el consentimiento informado. Después se dividió el grupo en ocho subgrupos, para poder efectuar la aplicación del instrumento. Una vez aplicado el instrumento se procedió a revisar cada uno de los cuestionarios para verificar que hubiesen sido respondidos en su totalidad, luego se sistematizó la información, y analizaron estadísticamente los datos mediante el SPSS20. Por último se analizó la información y se realizó el informe.

\section{Resultados}

Para identificar las actitudes de los estudiantes inicialmente se establecieron los puntajes para los rangos muy bajo, bajo, neutro, alto y muy alto. En la tabla 3 se presenta los puntajes correspondientes a cada rango, tanto para la prueba en su totalidad como por componente.

Tabla 3. Puntajes, categoría y rangos para determinar la actitud para la totalidad de la prueba y por componente.

\begin{tabular}{|c|c|c|c|c|c|c|c|c|}
\hline \multirow[b]{3}{*}{ Categoria } & \multirow{2}{*}{\multicolumn{2}{|c|}{ Total prueba }} & \multicolumn{6}{|c|}{ Dimensiones } \\
\hline & & & \multicolumn{2}{|c|}{ Cognitiva } & \multicolumn{2}{|c|}{ Afectiva } & \multicolumn{2}{|c|}{ Conductual } \\
\hline & $\begin{array}{c}\text { Mínimo } \\
\text { esperado } \\
61\end{array}$ & $\begin{array}{c}\text { Máximo } \\
\text { esperado } \\
305\end{array}$ & $\begin{array}{c}\text { Mínimo } \\
\text { esperado } \\
33\end{array}$ & $\begin{array}{c}\text { Máximo } \\
\text { esperado } \\
165\end{array}$ & $\begin{array}{c}\text { Mínimo } \\
\text { esperado } \\
15\end{array}$ & $\begin{array}{c}\text { Máximo } \\
\text { esperado } \\
75\end{array}$ & $\begin{array}{c}\text { Mínimo } \\
\text { esperado } \\
13\end{array}$ & $\begin{array}{c}\text { Máximo } \\
\text { esperado } \\
65\end{array}$ \\
\hline Muy bajo & & 61 & & 33 & & 15 & & 13 \\
\hline Bajo & 62 & 122 & 34 & 66 & 16 & 30 & 14 & 26 \\
\hline Neutro & 123 & 183 & 67 & 99 & 31 & 45 & 27 & 39 \\
\hline Alto & 184 & 244 & 100 & 132 & 46 & 60 & 40 & 52 \\
\hline Muy alto & 245 & 305 & 133 & 165 & 61 & 75 & 53 & 65 \\
\hline
\end{tabular}

Fuente: Los autores

Estos datos permitieron identificar el nivel de la actitud de los participantes para la investigación, tanto para la prueba total como por componente. En esta investigación se determinó como criterio para identificar el nivel de actitud la media. La actitud general de los participantes frente la investigación, se visualiza en la tabla 4 :

Tabla 4.Actitud de los participantes frente la investigación.

\begin{tabular}{ccc}
\hline $\mathrm{n}$ & Media & Desviación estándar \\
\hline 233 & 235.45 & 21.330 \\
\hline
\end{tabular}

Fuente: Los autores

La media obtenida se encuentra en la categoría alta, lo que permite afirmar que los estudiantes presentan una actitud positiva frente a la investigación.
En lo concerniente a los componentes de las actitudes considerados, los resultados se presentan en la tabla 5.

Tabla 5. Medias en cada componente de las actitudes

\begin{tabular}{cccc}
\hline Componente & $\mathrm{n}$ & Media & Desviación. estándar \\
\hline Cognitivo & 233 & 134.45 & 11.830 \\
Afectivo & 233 & 53.20 & 7.862 \\
Conductual & 233 & 47.79 & 6.323 \\
\hline
\end{tabular}

Fuente: Los autores 
Los resultados evidencian que los participantes tienen una actitud cognitiva muy alta y alto en el componente afectivo y en el comportamental. Según estosdatos la actitud más alta corresponde al conocer acerca del proceso de investigar, pero hay cierto nivel de correspondencia, entre el gusto e interés como por el realizar investigaciones.

La distribución de los participantes en cada opción de respuesta se presenta en la tabla 6 .

Tabla 6. Actitud de los participantes en las diversas opciones de respuesta por componente

\begin{tabular}{ccccccccccccc}
\hline & \multicolumn{11}{c}{ Opción de respuesta } \\
\cline { 2 - 13 } Dimensión & \multicolumn{1}{c}{ Muy baja } & \multicolumn{1}{c}{ Baja } & \multicolumn{1}{c}{ Neutra } & \multicolumn{1}{c}{ Alta } & \multicolumn{3}{c}{ Muy alta } \\
& $\mathrm{n}$ & $\%$ & $\mathrm{n}$ & $\%$ & $\mathrm{n}$ & $\%$ & $\mathrm{n}$ & $\%$ & $\mathrm{n}$ & $\%$ & Total \\
\hline Cognitiva & 0 & .0 & 0 & .0 & 1 & .4 & 92 & 39.5 & 140 & 60.1 & 233 \\
Afectiva & 0 & .0 & 2 & .9 & 34 & 14.6 & 158 & 67.8 & 39 & 16.7 & 233 \\
Comportamental & 0 & .0 & 1 & .4 & 18 & 7.7 & 162 & 69.5 & 52 & 22.3 & 233 \\
\hline
\end{tabular}

Fuente: Los autores

La tabla deja ver que los estudiantes universitarios en mayor proporción puntúan en la dimensión cognitiva, con una actitud muy alta $(60,1 \%)$, seguidos, en menor proporción, por quienes tienen una actitud alta $(39,5 \%)$. Entre tanto en la dimensión afectiva, los estudiantes se identifican en mayor proporción con una actitud alta $(67,8 \%)$, seguidos por quienes presentan una actitud muy alta $(16,7 \%)$ y neutra $(14,6 \%)$. En la dimensión conductual, al igual que en la afectiva, los estudiantes se identifican con una actitud alta $(69,5 \%)$, seguidos, por quienes presentan una actitud muy alta $(22,3 \%)$ y neutra $(7,7 \%)$.En general, puede señalarse de acuerdo con las tablas, la homogeneidad de los datos, que indican que la tendencia, tanto en la calificación general, como en las dimensiones, es que los estudiantes tengan una actitud alta frente a la investigación.

Para identificar las diferencias de las puntuaciones de las dimensiones según el semestre que cursan los estudiantes, se realizó una ANOVA de un factor. En la tabla 7 se presentan los resultados.

Tabla 7. Resultados prueba ANOVA para el factor nivel de formación y puntuaciones obtenidas en la escala de actitud.

\begin{tabular}{llccc}
\hline Componente & & gl & F & Sig. \\
\hline Cognitivo & Entre grupos & 9 & 1.807 & 0.068 \\
Afectivo & Entre grupos & 9 & 2.791 & 0.004 \\
Conductual & Entre grupos & 9 & 3.347 & 0.001 \\
\hline
\end{tabular}

Nivel de significancia $\leqslant 0.005$

Fuente: Los autores
Según estos datos, las diferencias entre puntuaciones obtenidas por los estudiantes en las dimensiones de la escala por semestre que cursan, se presentan como estadísticamente significativas en las actitudes afectiva (sig $0.004)$ y conductual $(0.001)$.

En la tabla 8 se indica entre que semestres se presentan diferencias en las puntuaciones obtenidas por los estudiantes en las dimensiones afectiva y conductual.

Tabla 8. Comparaciones múltiples (Bonferroni) para el factor semestre/asignatura y puntuaciones obtenidas en la aplicación de la escala de actitud hacia la investigación.

\begin{tabular}{lcccc}
\hline Componente & $\begin{array}{c}\text { (I) Nivel de } \\
\text { formación }\end{array}$ & (J) Semestre & $\begin{array}{c}\text { Diferencia de } \\
\text { medias (I-J) }\end{array}$ & Sig. \\
\hline \multirow{2}{*}{ Afectiva } & $2^{\circ}$ & $4^{\circ}$ & $-0.677^{*}$ & 0.002 \\
\cline { 2 - 5 } & $2^{\circ}$ & $7^{\circ}$ & $-0.552^{*}$ & 0.020 \\
\hline \multirow{2}{*}{ Conductual } & $9^{\circ}$ & $-0.603^{*}$ & 0.016 \\
\cline { 2 - 5 } & $3^{\circ}$ & $9^{\circ}$ & $-0.552^{*}$ & 0.013
\end{tabular}

Nivel de significancia $\leqslant 0.005$

Fuente: Los autores

Las diferencias estadísticamente significativas en las puntuaciones del componente afectivo se presentan entre los semestres II y IV y II y VII, siendo en ambos casos menorla puntuación en el II nivel. Con respecto al componente conductual, las diferencias estadísticamente significativas en las puntuaciones se presentan entre los semestres II y IX y III y IX, siendo menor la puntuación en los semestres II y III. 
Para el factor edad, la tabla 9 indica, a partir del ANOVA las diferencias en las puntuaciones de las dimensiones de la escala de actitud frente a la investigación obtenidas de los estudiantes.

Tabla 9. Resultados ANOVA para el factor edad de los estudiantes y puntuaciones obtenidas en la aplicación de la escala de actitud hacia la investigación.

\begin{tabular}{ccccc}
\hline Variable dependiente & & gl & F & Sig. \\
\hline Cognitiva & $\begin{array}{c}\text { Entre } \\
\text { grupos }\end{array}$ & 2 & 3.624 & 0.028 \\
\hline Afectiva & $\begin{array}{c}\text { Entre } \\
\text { grupos }\end{array}$ & 2 & 1.260 & 0.286 \\
\hline Conductual & Entre grupos & 2 & 5.005 & 0.007 \\
\hline
\end{tabular}

Nivel de significancia $\leqslant 0.005$

Fuente: Los autores

Según los resultados, las diferencias entre puntuaciones obtenidas por los estudiantes en las dimensiones de la escala por rangos de edad, se presentan como estadísticamente significativas en las actitudes cognitiva (sig 0.028) y conductual $(0.007)$.

En la tabla 10 se señala entre que rangos de edad se presentan diferencias en las puntuaciones obtenidas por los estudiantes en las dimensiones cognitiva y conductual.

Tabla 10.Comparaciones múltiples para el factor edad de los estudiantes y puntuaciones obtenidas en la aplicación de la escala de actitud hacia la investigación.

\begin{tabular}{lcccc}
\hline Componente & Edad & Edad & $\begin{array}{c}\text { Diferencia de } \\
\text { medias }\end{array}$ & Sig. \\
\hline Cognitivo & $15-20$ & $21-25$ & $-0.132^{*}$ & 0.023 \\
\hline Conductual & $15-20$ & $21-25$ & -0.215 & 0.006 \\
\hline
\end{tabular}

Nivel de significancia $\leqslant 0.005$

Fuente: Los autores

Las diferencias estadísticamente significativas en las puntuaciones de la dimensión cognitiva se presentan entre los rangos de edad 15 a 20 años y 21 a 25 años (sig 0.023), siendo menor la puntuación para el rango de 15 a 20 años. Con respecto a la dimensión conductual, las diferencias estadísticamente significativas en las puntuaciones, al igual que en la dimensión cognitiva, se presentan entre los rangos 15 a 20 años y 21 a 25 años (sig 0.006), siendo menor la puntuación para el rango de 15 a 20 años.

La tabla 11 evidencia, a partir de la prueba t de Student para igualdad de medias, las diferencias de las puntuaciones de las dimensiones según el género de los estudiantes.
Tabla 11. Prueba de muestras independientes para el factor género de los estudiantes y puntuaciones obtenidas en la aplicación de la escala de actitud hacia la investigación

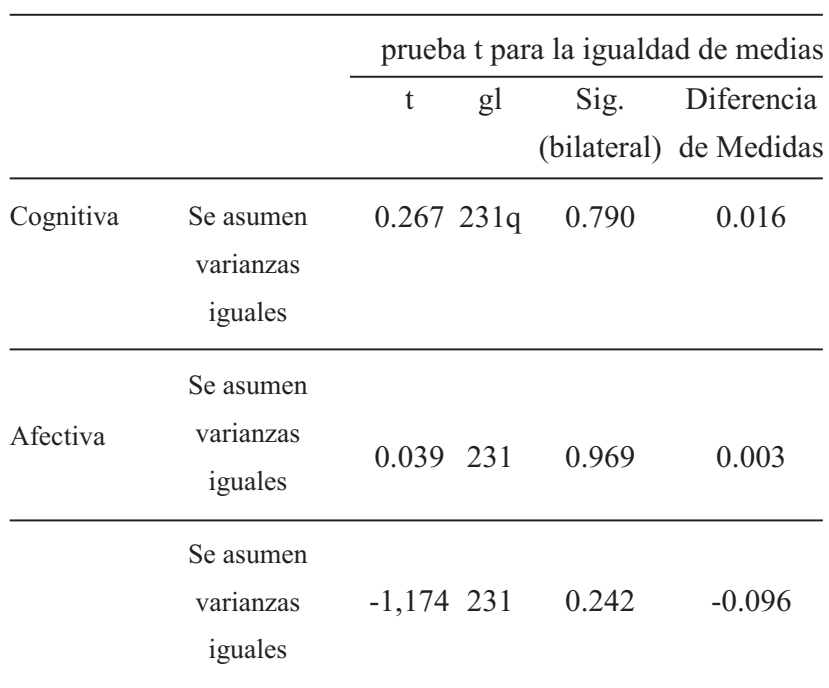

Nivel de significancia $\leqslant 0.005$

Fuente: Los autores

Los resultados permiten señalar que el género de los estudiantes no es un factor determinante en las diferencias que se presentan en las puntuaciones obtenidas en las dimensiones de la escala actitud frente a la investigación $(\operatorname{sig}>0,05)$.

\section{Comentarios}

Los datos encontrados en el presente estudio muestran respecto a variables sociodemográficas como edad $\mathrm{y}$ género, que la actitud hacia la investigación se incrementa a medida que los estudiantes tienen más años, dato que contrasta con los alcanzados por Rojas, Méndez y Rodríguez (2012) quienes detectaron que "variables de composición como la edad, el sexo, el semestre y el rendimiento académico del estudiante, no presentaron una asociación significativa con la actitud investigativa" (p.221) y Asimismo por Rojas (2009) quien evidencio que "aspectos como la edad, el sexo y el promedio académico no constituyen variables de correlación en casi ningún ítem examinado, lo que equivale a decir que estos no tienen un efecto importante" en la autoevaluación sobre la actitud investigativa (p.378).

Según lo anterior, no existirían diferencias en la actitud hacia la investigación entre los estudiantes universitarios según su edad, es decir ésta no determinan el interés por realizar trabajos de carácter científico, resultados que no coinciden con lo encontrado en esta investigación, que detectó menor actitud en los participantes más jóvenes, es posible que este resultado se encuentre más relacionado con los semestres cursados. Los participantes más jóvenes posiblemente sean los que inician carrera por tanto no han tenido un mayor acercamiento al proceso investigativo, lo que si ocurre con los que se encuentran en los siguientes 
rangos de edad y formación.En cuanto la variable género, los resultados indican que no se detectaron diferencias significativas, dato que coinciden con lo encontrado por Rivera y Torres (2006) quienes indagando acerca de la percepción que los estudiantes universitarios tienen sobre sus habilidades de investigación, encontraron resultados similares.

En lo concerniente al otro aspecto que se abordó, cómo se puntúa en los ítems, a continuación se resalta los que obtuvieron puntajes más significativos: en el ítem 8 , cuya afirmación dice, "la investigación requiere el hábito de leer" obtuvo una puntuación muy alta de $72,5 \%$, resultado que coincide con los obtenidos por Aceituno, et al, (1998, p. 42) en un estudio realizado con estudiantes de medicina, donde el $69.1 \%$ consideraron importante leer continuamente publicaciones científicas. El puntaje obtenido en el ítem 8 evidencia la importancia que los estudiantes dan a la formación del hábito de la lectura, ya que a partir de este se facilita la búsqueda de información para argumentar y comprender investigaciones, y asimismo, contribuye a la actualización en los avances científicos.

En el ítem 12, cuya afirmación indaga si los estudiantes utilizan el métodos científicos para acceder al conocimiento", y el $49.4 \%$ dicen que sí, deje entrever que el $51.6 \%$ consideran que existen otros métodos para acceder al conocimiento pero no que desconozcan o demeriten el método científico, sino que piensan que existen otras formas para conocer.

En el ítem 20, que dice "para hacer investigación es necesario tener motivación propia", el resultado arrojó una puntuación muy alta, con un $67,8 \%$, el cual es más alto que el encontrado por Aceituno et al. (1998, p. 44) evidenciaron que un $51.4 \%$ de los estudiantes manifestaron estar motivados para realizar trabajos científicos. Cuando Aceituno et al (1998, p. 44) analizan por que el 49,6\% de los estudiantes encuestados están poco motivados para trabajar en investigación, creen que se debe a "la escasa colaboración y apoyo docente al ejercicio propio de los estudiantes, a quienes no se les indican líneas o proyectos donde el alumno podría verter su interés", a diferencia de los resultados obtenidos en los estudiantes de Psicología donde se realizó este trabajo donde a través de una línea de investigación y el apoyo de los docentes, los estudiantes van desarrollando habilidades investigativas, evidenciándose un incremento en actitud positiva hacia la investigación a medida que avanza en los semestres.

Enel ítem 21, "la investigación es una actividad fascinante", el 43,3\%, de los participantes puntuó alto, resultado que coincide con los de Díaz, et al (2008, p. 11) quienes hallaron que el $72.5 \%$ de los encuestados afirmaron que les agrada investigar. A partir de dichos resultados se deduce que los estudiantes que participaron en este trabajo tienen una actitud positiva frente al ejercicio de investigación, la cual consideran una actividad interesante.
En el ítem 40 "la investigación no debería incluirse en los planes de estudio" obtuvo una puntuación de 52,4\%, lo que muestra que cerca de la mitad de los participantes consideran que investigar no debe hacer parte del plan de estudio, cantidad que se considera alta para el programa donde se realizó la investigación, teniendo en cuenta que en el plan de estudio de la carrera en la universidad estudiada tiene 10 asignaturas directamente relacionadas con ello. En relación a lo anterior se logra inferir que los estudiantes consideran que la inclusión de asignaturas relacionadas directamente con la investigación limita la actividad investigativa, al desarrollarse de forma simultánea a esta. Es posible que los estudiantes consideren la investigación"una tarea complementaria, relegada a último plano, después de las asignaturas y la recreación" (Aceituno, et al, 1998, p.44).

En lo concerniente a la pregunta de investigación que se interroga sobre las actitudes de los estudiantes sobre la investigación, los resultados muestran lo siguiente:

Respecto al Componente Cognitivo se encontró una actitud "muy alta", específicamente para los semestres IV y $\mathrm{X}$, seguidos, en menor proporción, por el componente afectivo puntuado en la categoría "alta" en los semestres VII, VIII y IX, y el componente conductual en los semestres I, II, III, V, VI y VII. En cuanto al componente cognitivo que mide lo que el sujeto sabe o cree saber sobre del objeto de la actitud, incluye ítems como: "la investigación aporta conocimiento nuevo", "investigación y creatividad están relacionadas", "la investigación requiere el hábito de leer", "la investigación contribuye al avance de la ciencia", "la investigación tiene reconocimiento social", "la investigación contribuye a resolver problemas actuales", “investigación, estudiantes de psicología y proyección social están relacionadas"; "la investigación requiere capacidad de síntesis". En este caso, indicaría que los estudiantes tienen una actitud favorable y poseen claridad acerca de lo que implica el ejercicio de investigar desde su proceso de formación en la universidad.

En lo referente a la actitud más baja de acuerdo al semestre se encontró que los estudiantes de II y V puntuaronbajo en el componente afectivo, el cual incluye ítems tales como: "Prefiero trabajar en equipo", "considero que la investigación es una carga para el estudiante", "me gusta leer y escribir", "la investigación me resulta fascinante", "me gusta el trabajo metódico", "la investigación me parece complicada", "la investigación me genera ansiedad", "la investigación me aburre", "la investigación me resulta pesada en los planes de estudio", "la investigación es una actividad frustrante".

En el III semestre el componente con puntuación más baja fue el conductual. Algunos ítems relacionados a esta dimensión son: "Con frecuencia busco información”, "me capacito en forma permanente", "tengo en cuenta las normas morales y éticas", "corroboro el conocimiento por mí mismo", "observando la realidad se me ocurren ideas para investigar", "leo informes de investigación", "me esfuerzo por comprender procesos investigativos", "por lo 
general pongo a consideración mis ideas", "evaluó investigaciones con alguna frecuencia".

Como conclusiones es importante resaltar que los estudiantes presentan una mejor actitud frente a la investigación a medida que avanzan en sus estudios, reflejando una mayor implicación frente al ejercicio investigativo, En relación a lo anterior, Díaz, et al, (2008, p. 11) encontraron en los estudiantes de Medica de distintas universidades del Perú "que el nivel de conocimientos guarda relación directa con el año de estudios". A diferencia del estudio de Shrigley (Citado por Vásquez \& Manassero, 1995, p. 339), donde señalan que "la actitud positiva hacia las ciencias decrece a medida que el estudiante progresa en sus estudios hacia niveles superiores, coincidiendo con otros estudios, incluso entre estudiantes especializados en ciencias". La tendencia de los estudiantes de Psicología con base en la puntuación total de la escala, es una actitud alta $(65,7 \%)$, o muy alta $(33,9 \%)$ frente a la investigación.

\section{Referencias}

Aceituno, H., Lanz, J., Lanz, J. \& Lander, G. (1998). Interés, conocimientos, recursos y limitaciones de los estudiantes de medicina en la investigación científica. Gaceta Medica de Caracas, 106(1), 40-46.

Aldana, G. \& Joya, N.(2011). Actitudes hacia la investigación científica en docentes de metodología de la investigación. Tabula Rasa, 14, 295-309.

Chaparro, L. (2004). Actitudes hacia la ciencia y la investigación en estudiantes del programa de Administración de Negocios internacionales de la Universidad de la Sabana. Manuscrito inédito, Departamento de Gestión para el Desarrollo Humano, Universidad de la Sabana, Chía, Colombia.

Díaz, C., Manrique, M., Galán, E. \& Apolaya, M. (2008). Conocimientos, actitudes y prácticas en investigación de los estudiantes de pregrado de facultades de medicina del Perú. Acta médica. Peruana, 25 (1),9-15.

Jiménez, W. (2006). La formación investigadora y los procesos de investigación científico-tecnológica en la Universidad Católica de Colombia. Studiositas 1 (1), 36-43.

Kaan, Z. \&Göksu, G. (2010). La investigación de factores que afectan la actitud de los estudiantes universitarios hacia la participación en la investigación científica. Procedía Social and Behavioral Sciences, 2, 5183-5189.
Nobigrot-Kleinman, D., Nobigrot-Streimbleinsky, M., \&Galván-Huerta, S. (1995). Las actitudes hacia la investigación y el aprendizaje en estudiantes de Medicina, UNAM: 1984-1994. (1995). Salud Pública de México, 37, 316-322.

Piña, R. \& León, R. (2013). Caracterización del proceso de formación investigativa del estudiante universitario de tecnología de la salud. Recuperado 20 de noviembre de 2015 de

http://www.bvs.sld.cu/revistas/san/vol18_3_14/san18314. htm

Rivera, M. \& Torres, C. (2006). Percepción de los estudiantes universitarios de sus propias habilidades de investigación. Revista de la comisión de investigación de FIMPES, 1,(1), 36-49.

Rojas, H. (2009). Formar investigadores e investigadoras en la universidad: Optimismo e indiferencia juvenil en temas científicos. Revista Latinoamericana de Ciencias Sociales, Niñez y Juventud, 7 (2), 1595-1618.

Rojas, H., Méndez, R. \& Rodríguez, A. (2012). Índice de actitud hacia la investigación en estudiantes del nivel de pregrado. Entramado, 8(2), 216-229

Valente G., S. y Viana L., De O. (2007). El pensamiento crítico-reflexivo en la enseñanza de la investigación en Enfermería: Un desafío para el profesor. Revista de Enfermería Global, 10, 1-8.

Vásquez, A., \&Manassero, M. (1995). Actitudes relacionadas con la ciencia: Una revisión conceptual. Investigación y Experiencias Didácticas, 13, 337-346.

\section{ANEXO 1}

CUESTIONARIO PARA CONOCER LAS ACTITUDES DE LOS ESTUDIANTES DE PSICOLOGÍA DE UNA UNIVERSIDAD PRIVADA DE LA CIUDAD DE BUCARAMANGA FRENTE A LA INVESTIGACIÓN. DATOS SOCIODEMOGRÁFICOS 
Edad

Género

Asignatura

Nivel de formación

A continuación encontrará una serie de enunciados asociados a la investigación científica (Adaptados de: Aldana y Joya, 2011); señale con una $X$ en la columna correspondiente la respuesta con la cual se sienta identificado. No piense mucho su respuesta. No hay respuestas buenas ni malas.

1. Muy en desacuerdo

4. De acuerdo
2. En desacuerdo

5. Muy de acuerdo
3. Ni de acuerdo ni en desacuerdo

EJEMPLO

\begin{tabular}{|c|l|c|c|c|c|c|}
\hline $\mathbf{N}^{\circ}$ & Ítem & $\mathbf{1}$ & $\mathbf{2}$ & $\mathbf{3}$ & $\mathbf{4}$ & $\mathbf{5}$ \\
\hline 1 & Investigar es un ejercicio dificil & & & & $x$ & \\
\hline
\end{tabular}

Al escoger la opción No.4, la persona está de acuerdo con que investigar es un ejercicio difícil.

\begin{tabular}{|c|c|c|c|c|c|c|}
\hline$N^{o}$ & Item & 1 & 2 & 3 & 4 & 5 \\
\hline 1 & Prefiero trabajar en equipo. & & & & & \\
\hline 2 & Me gusta buscar información. & & & & & \\
\hline 3 & La investigación es una carga para el estudiante & & & & & \\
\hline 4 & La investigación aporta conocimiento nuevo. & & & & & \\
\hline 5 & Me gusta escribir sobre investigación & & & & & \\
\hline 6 & Investigación y creatividad están relacionadas. & & & & & \\
\hline 7 & Me preparo en forma permanente & & & & & \\
\hline 8 & La investigación requiere el hábito de leer. & & & & & \\
\hline 9 & La investigación contribuye al avance de la ciencia. & & & & & \\
\hline 10 & La investigación tiene reconocimiento social. & & & & & \\
\hline 11 & La investigación contribuye a resolver problemas actuales & & & & & \\
\hline 12 & Utilizo métodos científicos para acceder al conocimiento. & & & & & \\
\hline 13 & Investigación, estudiantes de psicología y proyección social no tienen relación. & & & & & \\
\hline 14 & La investigación es importante porque resuelve problemas sociales. & & & & & \\
\hline 15 & La Investigación requiere capacidad de síntesis. & & & & & \\
\hline 16 & La investigación contribuye a detectar errores de la ciencia. & & & & & \\
\hline 17 & La investigación sólo confirma lo que ya se sabe. & & & & & \\
\hline 18 & La investigación contribuye a corregir errores del sentido común. & & & & & \\
\hline 19 & La investigación facilita la interacción entre disciplinas. & & & & & \\
\hline
\end{tabular}




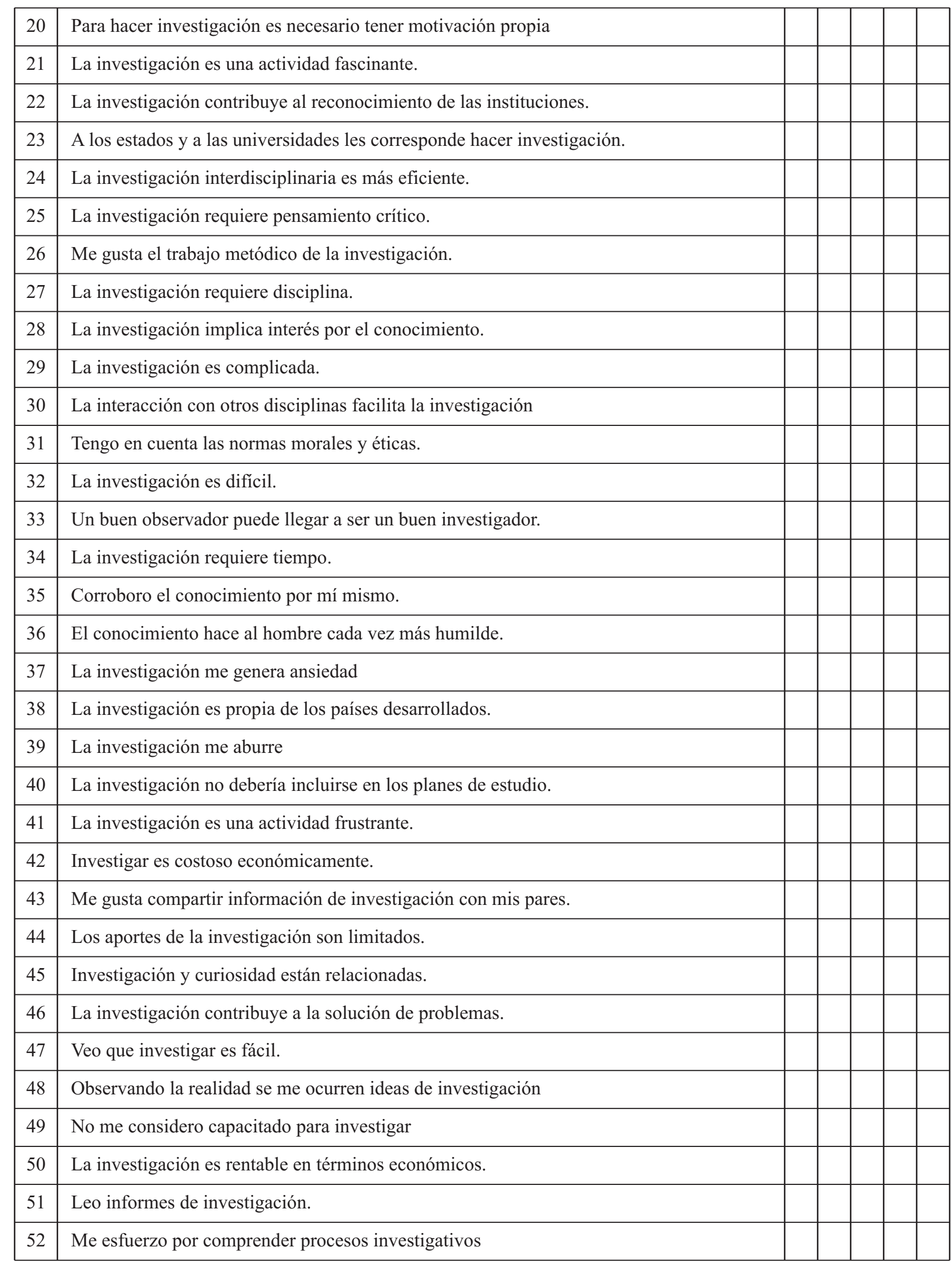




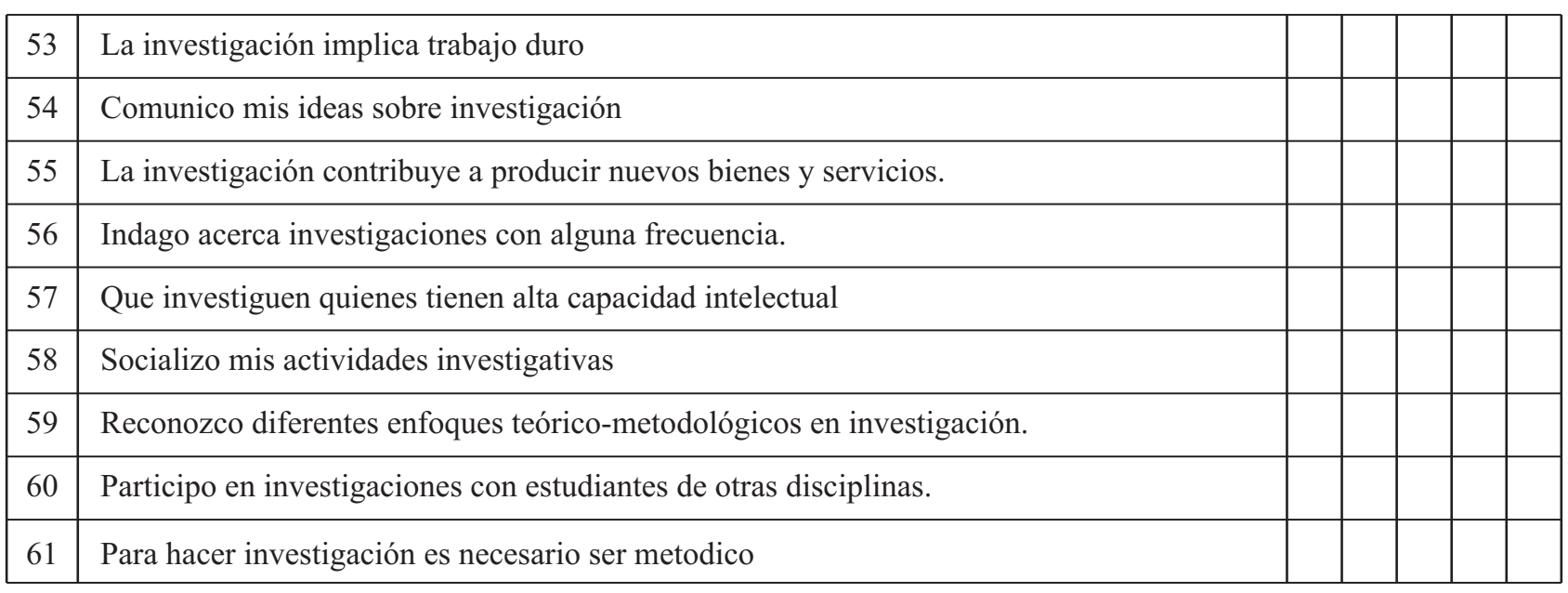

